\title{
FORGING-LIKE ROLLING WITH HIGH REDUCTION OF SBQ LONG PRODUCTS*
}

\author{
Francesco Toschi ${ }^{1}$ \\ Alberto Lainati ${ }^{2}$ \\ Giuseppe Muscarà ${ }^{3}$
}

\begin{abstract}
The Special Bar Quality family comprises a wide range of steel grades, mainly requested by the automotive, energy and engineering sectors, for applications where significant performances are required (eg. stresses, temperatures, working cycles, etc.). To meet these requirements it is necessary to reach a precise control of both the mechanical characteristics and metallurgy structures along the whole production chain. For semi-product with large dimensions, such as equivalent diameters around $300 \mathrm{~mm}$, this is traditionally obtained through a manufacturing route which comprises the casting in ingots followed by reheating, forging and rolling. After 2010, new processes were investigated and technologies developed to replace the ingot route with a continuous casting followed by controlled high-reduction rolling. In these processes a seamless integration of CCM with rolling is implemented so to obtain the precise control of material flow, applied deformation and working temperatures. By using the proper combination of rolling diameter, speed and applied reduction, a very good control of the metallurgy structure across the whole section can be obtained, so to also remove the internal discontinuities ("voids") generated during the continuous casting. These processes, which can be defined "forging-like", are applicable to several grades also with large semi-product dimensions (300 mm and more). Besides, removing the ingot route means increased yield and productivity, which is very beneficial to the economical management of the plant. An example of this "forging-like" process is represented by the high-reduction blooming stand installed by Acciaierie Venete in the Padova-Camin mill. This article describes its main concepts as well as its technology and process features.
\end{abstract}

Key-words: SBQ; rolling; forging; voids; metallurgy microstructure

Technology - Primetals Technology Italy

R\&D - Primetals Technology Italy

Sales - Primetals Technology Italy 


\section{INTRODUCTION}

For several years, the steel market has been disrupted by the financial and industrial crisis that has afflicted the world globally and the over-production capacity is now endemic, with no signs of relief in the short or medium term. As a consequence, the emphasis has shifted from increasing production capacity to product quality and optimization of processing costs. This obliges producers to seek improvements of flexibility and of production processes. In addition, the increased market competitiveness in different sectors leads to a growing demand for quality.

Specifically, for the steel production, the main driving forces are represented by mechanical constructions, in particular the automotive industry, and by the energy sector. As regards the automotive industry, the main players of the sector have assembly lines distributed worldwide, which must meet the most recent manufacturing standards. With respect to the energy sector, its development in remote locations requires durable performance over time and mechanical members of large size (e.g. the impressive drive shafts). In addition, very strict rules govern the construction and maintenance of nuclear power plants, whose components must ensure performance and reliability under severe operating conditions.

It is precisely in these sectors, that high-quality steel long products (Special Bar Quality or SBQ) are mainly use. These products require high quality, dimensional tolerances, surface finish, mechanical and microstructural characteristics, in order to provide the desired performance in severe operations conditions (e.g. stresses, temperatures, durations of cycles, etc.). In particular, in case of large dimensions, such as above about 200-300 mm equivalent finished diameter, the real performance of the final product depends on all the construction steps of the product itself, that influence each other, and that, therefore, must be integrated along the entire production chain from steelworks to rolling. As regards the semi-finished long products of large dimensions, such as above about 200-300 mm finished diameter, besides the traditional way of ingot casting followed by forging, there are different solutions that provide the continuous casting followed by rolling prearranged according to an appropriate strategy.

\section{CONCEPTS}

During the continuous or ingot casting, the different cooling speeds in the section induce inhomogeneity of solidification conditions, with shrinkages and release of gases dissolved in the product matrix, leading to undesirable phenomena of discontinuity in the heart ("voids"). The number and extent of these phenomena increase with the size of the product. Such voids can severely affect the performance of the quality finished product, and therefore it is essential to ensure their complete closure.

To date (2017), in case of large products, this is mainly obtained with a processing cycle which includes ingot casting followed by forging. This cycle is undoubtedly effective thanks to the action of the centripetal-compressive forces applied from the forging, but involves high costs of investment and operation.

New rolling technologies are available, where billets and blooms are continuously cast, and then rolled with a suitable combination of high reduction and roll diameter. These innovative rolling processes are generically called forging-like, as they may be compared to the forging process. Indeed, they allow to obtain a closure of the voids and the control of metallurgical matrix on the entire section of the product. 
The plastic deformation imprinted during rolling is different from the one made by forging, and the exerted forces are such that the material flow takes place more on the outside of the bar than in its heart. In order to increase the penetration of the deformation and to ensure that the deformation acts effectively up to the center of the structure, it is necessary to increase both the values of reduction and of the rolls diameters, without, however, tear or damage the skin of the product. Therefore, it is necessary to define a precise rolling strategy that allows to obtain a satisfactory closure of the heart discontinuity, thus promoting the integrity and uniformity of the product structure for subsequent processing.

It is also essential to have control of all process variables (mechanical, thermal and metallurgical) through all production stages: melt shop, continuous casting and rolling mill.

The continuous casting and the forging-like rolling make it possible to have higher productivity, higher metallic yield and a better surface quality, than the ingot cycle. In addition, time of production change is shortened, thus favoring the operational flexibility. Hence, it can be argued that the processing costs are lower and that the profitability of the overall operations is improved, even for limited production lots.

\section{CUSTOMER REQUIREMENTS}

At a primary Italian special steel producer, mainly catering to the automotive industry, the main purpose of the new blooming mill was to roll higher reductions of the cross section, thus improving the metallurgical properties of the final product and reducing processing costs. Since the installation of the new blooming was made in an existing plant in operation, there was the necessity to minimize the interference and the interruptions in the existing production, during all the phases of the project realization, from civil works to installation and commissioning.

\section{PARTNER OF PRIMETALS TECHNOLOGIES}

Primetals Technologies and Centro Sviluppo Materiali (RINA-CSM) have worked together to research, develop and industrialize this innovative process of highreduction rolling, called forging-like and applied to blooms produced by continuous casting.

In the field of long products, Primetals Technologies has carried out extensive research and experimentation to improve the process know-how and develop new technologies. Some examples are worth mentioning, such as the re-designed train areas to ensure the rolling stability also with thermo-mechanical process, the newly developed sizing group to ensure ultra-precision and free-sizing, various in-line applications for process management and quality control. In particular, in the roughing area, Primetals Technologies has a solid tradition of design, sale and installation of rolling mills for blooms for different applications, with about more than thirty plants realized, in housing or housingless design, in fixed or sliding type.

RINA-CSM, a technical center headquartered in Rome, has a long experience of research and experimentation in the field of materials for various applications. Its activities are mainly focused on the design of materials and products, optimization and development of manufacturing processes, metallurgical design of special steels and alloys, development of innovative pilot plants, special testing equipment and reduction of environmental impact. 


\section{RESEARCH ABOUT THE FORGING-LIKE PROCESS}

The research about the forging-like technology began with a careful study of the reference literature of academic institutions and industrial manufacturers. It has then moved into the definition and development of an integrated model that describes in detail all the mechanical (stress and strain), thermal (heating, cooling, latent heat as a result of phase transformations) and metallurgical (metallurgical phases and its components) conditions of the processes. This model has been tested, developed and finally validated in RINA-CSM laboratories.

The study was meant to verify that the appropriate combination of the reduction applied by the rolls, their diameter and the rolling speed, can result in an integrated continuous casting and rolling process with excellent results in the closure of voids. Another important objective was to ascertain that a product which meets the required standards can be obtained, with particular reference to quality steels for the automotive industry. The study was supported by appropriate FEM and numerical modeling platforms, that allowed to simulate the various processes, to understand in detail the influence of the parameters, define and optimize the rolling strategies. Firstly, the modeling results were verified and fine-tuned with trial protocols in a pilot plant. Secondly, the validation and consolidation of the parameters of the tested model were obtained and finally the model was integrated in the control automation of industrial plant. This model also allows the pre-production simulation for the definition of new rolling strategies, in case the need of rolling new dimensions and / or new grades arise.

To conclude, it was demonstrated that, in many cases, the manufacturing route of ingot and forging can actually be replaced by that of continuous casting and forginglike rolling. Undoubtedly, this route is cost-effective from various points of view, such as the layout simplification, the lower investment costs, the reduction of processing costs and the industrial operation streamlining. The first industrial application of the new forging-like technology took place in the new roughing mill of a primary Italian special steel producer.

\section{DEVELOPMENT AND TESTS}

In order to identify the correct rolling process and equipment, a careful analysis of the deformations had to be carried out and the parameters of a correct penetration of the deformation are to be considered. Indeed, the deformation is necessary to ensure both the high degree of internal integrity and the isotropy of the material. In addition, a correct rolling process must keep the surface intact, eliminating the insurgence of surface defects. In the forged product, these defects are eliminated during the subsequent processing phases, but in the rolled product are less tolerated because the post rolling processing needs to be minimized for low product costs. Therefore, during the FEM simulations the two most significant parameters (i.e. Tanaka and Nakasaki) were evaluated to define if a deformation process leads to the closure of possible heart discontinuities. The process sizing parameters were the given drafts (in total and per each rolling pass) and the diameter of the rolls. Speed and temperatures on the surface and across the product section influence the depth of penetration of the deformation and, thus, the capability to close the voids. In order to optimize the internal integrity, the damage index on the 2 main deformation directions was evaluated. 
Two products were considered: a cast round bloom with $800 \mathrm{~mm}$ diameter and a $705 \times 610 \mathrm{~mm}$ ingot, with 5 and 11 passes respectively, with draft up to $110 \mathrm{~mm}$. As regards the round, the total deformation in the heart changes from 0.4, for a 1060 $\mathrm{mm}$ roll diameter, to 0.6 for a $1300 \mathrm{~mm}$ diameter and the maximum difference across the section changes from 1.6 to 1.1 , thus showing better isotropy of the material with bigger rolls diameter. Considering the total closure of porosity on reference areas in the heart equal to 10, 25 and 35\%, it can be noted that with $1060 \mathrm{~mm}$ diameter, the Tanaka index does not change, while with $1300 \mathrm{~mm}$ diameter the index increases considerably. With the ingot simulation, two typical steels were considered: an AISI304 and a AISI H13 for utensils. The cross section thermal gradient was introduced in the FEM simulation in order to increase the penetration of the deformation. In the 11 pass sequence, the two indexes are consistent and confirm that, in this case, the porosity closure in the heart occurs from the 5th to 9th pass.

To conclude, a correct sequence must close the porosity completely (Nakasaki index lower than -0.2 and Tanaka equal to 100) and preserve the surface integrity in order to avoid costly subsequent processing. In the studied sequence the surface damage index is kept below the theoretical limit of 1, except from a pass where it gets to 1.2 in the corners. Rolling tests on three diameters were conducted, including a test on a $98.5 \mathrm{~mm}$ round for the evaluation the internal integrity and the microstructural isotropy on AISI304. The results met the expectations for this type of steels and diameters.

\section{DESCRIPTION OF THE SUPPLY}

In the new installation, it was necessary to carefully consider the flow of material from the continuous casting to the furnace and to the new blooming, up to the existing continuous mill area using automated procedures. The automatic check of the process ensures high efficiency and reduces the risks of human error. In the rolling process each bar is monitored by means of data provided to the operator and to the plant control system, which ensures the accuracy of details necessary to the continuity of the process.

Primetals Technologies' new blooming mill line starts at the exit of blooms caster, where a transfer device can quickly deposit the blooms on the reheating furnace inlet roller way or on the cooling bed. A walking beam device moves the blooms forward on the cooling bed, ensuring different cooling profiles depending on the products. A high-pressure descaler is installed between the furnace and the blooming mill. The blooming mill is designed as a reversible two-high sliding stand, which allows to change the groove maintaining the rolling centerline fixed. During the reversing operations, the product is moved forward by means of roller ways and electromechanical manipulators. The stand is equipped with a roll quick change device. The rolls have $1,800 \mathrm{~mm}$ table and $1,000 \mathrm{~mm}$ diameter. The stand processes about 110 tons per hour of round blooms with a maximum weight of 10 tons and diameters range between 350 and $600 \mathrm{~mm}$. The finished products are squares 180, 240, $280 \mathrm{~mm}$, and potentially even rounds with diameters between 180 and $315 \mathrm{~mm}$. A hydraulic shear cuts the product before the existing continuous mill, where the future installation of a high-efficiency induction heater has been considered.

The electrical supply is based on Siemens technology, well known for its excellent performance in rolling with variable loads. The scope of supply includes a full control and automation suite, including the medium-voltage transformers for the stand, the 
AC main controls with two $1800 \mathrm{kw}$ motors, the AC auxiliary, the automation level 1, the mechatronic components and a closed circuit television system.

Primetals Technologies was also responsible for erection, commissioning, and training of personnel. The contract was signed in 2012, the plant started the production in 2014 and in 2015 the final setup took place.

\section{ACHIEVED RESULTS}

The advantages of this new technology can be summarized as follows:

- increased market share in the quality long products sector

- dimensions

- grades

- metallurgical quality

- mechanical characteristics

- surface quality

\section{CONCLUSIONS}

The high quality long products (Special Bar Quality or SBQ) are used mainly in the mechanical sector, especially the automotive industry, energy and petrochemicals sectors. Such products are required to have high performance of dimensional tolerance, surface finish, mechanical and microstructural characteristics. The forginglike process of Primetals Technologies allows to eliminate the discontinuity in the heart or the voids produced during cooling after casting, with quality comparable to the forging process. Compared to the path of ingot and forging, the forging-like ensures higher productivity and the overall reduction of processing costs, thus making it suitable also in case of limited production lots.

\section{REFERENCES}

1 M. Nakasaki, I. Takasu and H. Utsunomiya, Application of hydrostatic integration parameter for free-forging and rolling, Journal of Material Processing Technology, 177 (2006)

2 Rahul Shashikant Nalawade, Prafull Prataprao Patil, G Balachandran, V Balasubramanian, FEM Simulation and Industrial Validation of Void Closure in a Large Cross-Section Hot Rolled Low-Alloy Steel, AISTech Conference, 2015 